\title{
Status of the LESIA High Contrast Bench development in the framework of the E-ELT
}

\author{
M. Mas ${ }^{1, a}$, P. Baudoz, R. Galicher, G. Rousset, J. Baudrand, C. Coton, and F. Assémat
}

LESIA, Observatoire de Paris-Meudon, CNRS and University Denis Diderot Paris 7, 5 place Jules Janssen, 92195 Meudon, France

\begin{abstract}
In the framework of the instrument Exo-Planet Imaging and Spectrograph (EPICS), an optical bench is being set up in the Laboratoire d'Etudes Spatiales et d'Instrumentation en Astrophysique (LESIA). With this bench, we will test a new high contrast technique, the Self-Coherent Camera (SCC). As already described in Galicher et al. (2008), the SCC is able to measure the incoming phase defects and, in the same time, detect a faint companion in the residual speckle field. To reach high contrast detection, both coronagraphy and adaptive optics are used to make measurements with the SCC. The principle of the instrument is based on interferences between two beams. One of them is spatially filtered using a pinhole which filters the high frequencies of the star beam and stops the light from the companion. The recombination of these two beams takes place at the pupil plane in a Fizeau type setup. As a result, the speckles in the final focal plane created by residual aberrations will be encoded with fringes. To test this instrument in laboratory environment, we are developing a test bench which will combine high performance coronagraph (four-quadrant phase mask coronagraph), an extreme adaptive optics (a Boston electrostatic deformable mirror with $32 \times 32$ actuators) and the SCC itself. After introducing the principle of the SCC, we show the first results of a numerical study. We eventually describe the status of the test bench development.
\end{abstract}

\section{Introduction}

Since the 90's, more than 300 exoplanets have been detected. Most of them were found using indirect detection studying radial velocimetry. However, to understand the formation of exoplanets, it is mandatory to detect directly photons from the planet, reaching a very high constrast and high angular resolution. In the framework of the instrument Exo-Planet Imaging Camera and Spectrograph (EPICS, Kasper et al. 2009 [1]), new instrumental concepts are necessary to improve the sensitivity of the telescope down to rocky planets. This concept will couple adaptive optics and coronagraphy. Nevertheless, even after a correction with an adaptive optic system, there still are residual static aberrations in the pupil plane. These defects appear as static or quasi-static speckles in the focal plane. An exoplanet located among these speckles is hardly detected even when using differential imaging techniques (Cavarroc et al. 2006 [2]).

We propose to use the Self-Coherent Camera (SCC) (Baudoz et al. 2006 [3], Galicher et al. 2007 [4]), to overcome this limitation. Its principle is based on the incoherence between the stellar and the companion lights. The purpose of that device is to discriminate, in a field of view, a companion image from the speckles created by wavefront defects on the plane of the detector. It couples coronagraphy and extreme adaptive optics. The SCC has two functions, on one hand, it estimates the phase. Thus, using a deformable mirror, we can correct the wavefront and increase the contrast. On the other hand it "recognizes" the image of the companion among a field of speckles. A prototype of SCC is being tested at LESIA in order to estimate the performance of the SCC.

\footnotetext{
a e-mail: marion.mas@obspm.fr
} 


\section{The Self-Coherent Camera}

\subsection{Principle}

In this section, we describe the principle of SCC by following the light of a star and its planetary companion. The telescope collects photons from both the star and its companion. The beam is divided in two parts by a beamsplitter. On one channel, a coronagraph centered on the stellar beam stops the photons from the star, when the ones of the planet are not affected. Because of optical aberrations, the rejection of the stellar light by the coronagraph is not perfect, hence there are some speckles from the star in the focal plane. The second channel is a reference beam which need to be coherent with the star only. For example, we can use a pinhole to filter high frequencies of the star beam wavefront and stop the companion light. Consequently, only light from the star goes through this filter. The pinhole diameter size allows us to ajust the light level in the reference channel. These two beams are optically brought in the same focal plane. Both beams interfer on the focal plane as in a Fizeau type interferometer. However, as the stellar and the companion lights are incoherent, photons from the planet are not encoded [3], [4]. As described in Galicher et al. 2007 [4], after a post-processing treatment, it is possible to discriminate the image of the planet from the speckles. With the SCC, we can also estimate the residual phase (Galicher et al. 2008 [5]). Then, using a deformable mirror upstream from the coronagraph, we can correct the wavefront. The SCC uses the same image for both the phase analysis and the exoplanet imaging channels, so, compared with a classical adaptive optic system, it has the advantage of having no differential aberrations. The principle of the SCC is shown in Fig. 1.

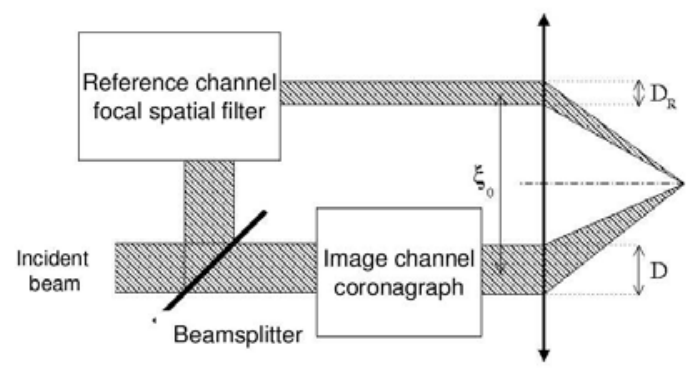

Fig. 1. Principle scheme of the SCC. The beams from the image channel containing photons from both the star and its companion (on the bottom) and from the reference with only photons from the stellar light (on the top) interfer like in a Fizeau interferometer on the focal plane. The photons from the companion are not encoded with fringes. With the SCC, we also can estimate the phase [5] and correct the wavefront with a deformable mirror.

\subsection{Numerical study : estimation of the phase and correction of the wavefront with a short exposure time}

We show, in this section, numerical simulations of the SCC coupled with a 32x32 actuators deformable mirror and a perfect coronagraph. The exposure time is shorter than the coherence time of the speckles which are static in this case. There is no photon noise. For this simulation, we assume we have a monochromatic light. We have simulated a static aberration upstream of the coronagraph with a 20 $\mathrm{nm}$ rms amplitude. In a first step, we estimate the residual phase, then, we correct the wavefront. In Fig. 2 (left), we show the radial profile with respect to the angular distance in $\lambda / \mathrm{D}$. The black curve represents the $5 \sigma$ detection in the focal plane with no SCC, no coronagraph and no correction. The pink curve is the $5 \sigma$ detection with the SCC and the coronagraph before correction. The image in the middle of the Fig. 2 corresponds to this pink curve. It represents the SCC image in the focal plane 
before correction, the speckles are spatially encoded. Then the red, green and blue lines are the $5 \sigma$ detections with the SCC and the coronagraph, after the first, the second and the third correction with the deformable mirror respectively. The SCC image after the third iteration of correction is on the right of Fig. 2. The dark square area is the effective zone of correction of the simulated deformable mirror. In this simulation, to estimate the phase, we linearise a non linear function [5], as a consequence, the estimation is not perfect and we have to do some iterations to correct the wavefront.
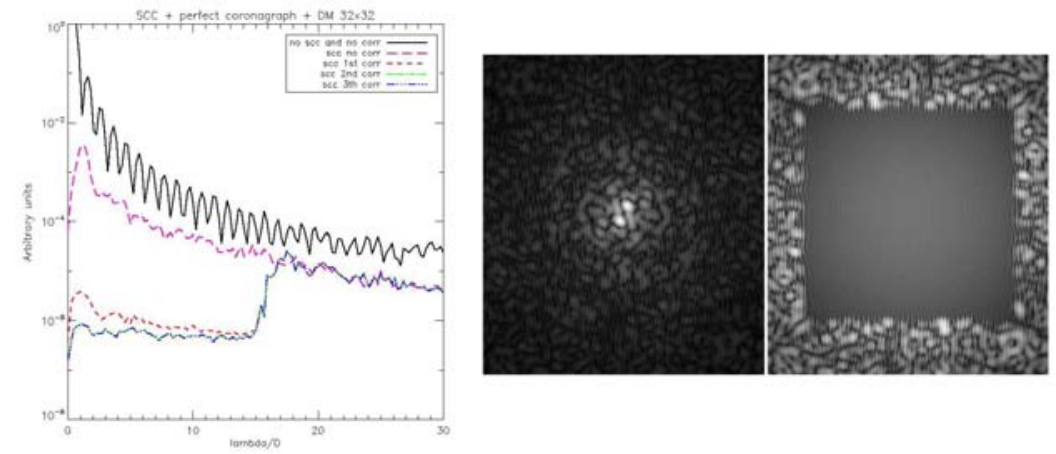

Fig. 2. Simulation of the SCC coupled with a $40 x 40$ deformable mirror, see the conditions in the text. On the left : the graph is the radial profile with respect to the angular distance in $\lambda / \mathrm{D}$. Black curve : $5 \sigma$ detection in the focal plane with no SCC, no coronagraph and no correction. Pink curve : $5 \sigma$ detection with the SCC and the coronagraph before correction. Red, green and blue lines : $5 \sigma$ detection with the SCC, the coronagraph, after the first, the second and the third correction with the deformable mirror. In the middle and on the right : it is the SCC image without correction and after 3 corrections respectively, with the coronagraph in both cases.

\subsection{Numerical study : estimation of the phase with a long exposure time}

On this section, we present some preliminary results of a simulation of a SCC working with a groundbased telescope. The exposure time is then longer than the coherence time of the turbulent speckles which are averaged. In this case, the residual speckles are quasi-static.

We simulate a turbulent atmosphere using a large phase screen, following Von Karman model (Consortini et al. 1973 [6]). We choose an outer scale which size is $L_{0}=20 \mathrm{~m}$, the wind speed is $v=5 \mathrm{~m} / \mathrm{s}$ and, at the observing wavelength $0.8 \mu \mathrm{m}$, the Fried parameter is $r_{0}=0.1 \mathrm{~m}$. We simulate an $8 \mathrm{~m}$ diameter telescope, one pixel on screen representing 0.1 meter $\left(\operatorname{size}_{\text {pix }}=0.1 \mathrm{~m}\right)$. This telescope is coupled with an extreme adaptive optic system with 40x40 actuators which corrects the turbulent phase with a very small delay. The temporal sampling is $S_{A O}=10^{-3} s$.

To simulate this adaptive optic system, we create a second phase screen from the first one with a shift of $n b_{p i x}=\frac{v . s_{A O}}{s i z e p i x_{i x}}=0.05$ pix. Then, using a circular mask, we cut the high frequencies in the Fourier domain of this second screen. We substract the second phase screen to the first one. As a result, on the final screen, the low frequencies are corrected, when the high ones are not. Now, on this corrected screen, we translate pixel by pixel a pupil with a diameter size of 80 pixels. All these phases represent a phase which evolves for each pixel, it is the dynamic phase.

The aim of the simualtion is to estimate the phase with the SCC during a long exposure time. One SCC image $\left(S C C_{i}\right)$ is defined from the phase $p h_{i}=p h_{d y n_{i}}+p h_{s t a t}$, with $p h_{d y n_{i}}$ the dynamic phase and $p h_{\text {stat }}$ a static one of $3 \mathrm{~nm}$ rms. This static phase corresponds to the static aberrations due to the optics on the bench. To simulate the long exposure time, we sum a serie of SCC images. We then average these images and we get a final SCC image. We estimate the phase from this averaged SCC image. We have done a simulation averaging up to 1000 SCC images corresponding to an exposure time of 20 secondes. The figure in Fig. 3 shows the root mean square of the difference between the static phase and the estimated one with respect to the number of SCC images. The more SCC images we take, the 
longer the exposure time is and the better the estimation of the static aberration is. The dynamic phase is apparently averaging, as expected, with the square root of the number of independant images. On the right, the 4 pictures represents from left to right, from top to bottom, the phase estimated from 40, 200, 1000 SCC images and the static phase itself. We clearly see linear structures in the images that come from our model of atmosphere which only takes into account one turbulent layer. This is a preliminary result of the SCC coupling with a ground-based telescope. More sophisticated simulation of the correction of the wavefront with a long exposure time is under study in the framework of the E-ELT instrument study. For more results with long exposure time, see also Baudoz et al. 2009 [7] in this volume.
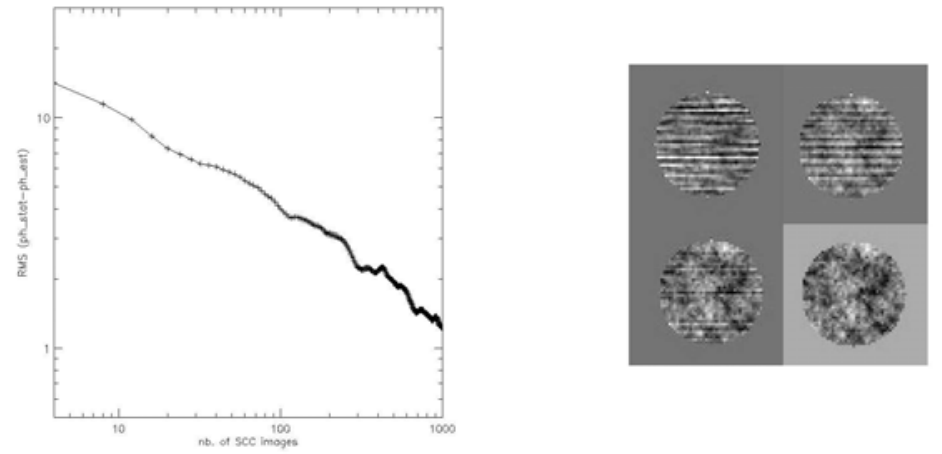

Fig. 3. On the left : the graph represents the root mean square of the difference between the static phase and the estimated one with the number of SCC images. On the right : from left to right, from top to bottom the 4 pictures represent the phase estimated from the average of 40,200 and 1000 SCC images and the static phase respectively.

\section{Status of the experiments}

\subsection{The High Contrast Imaging Bench}
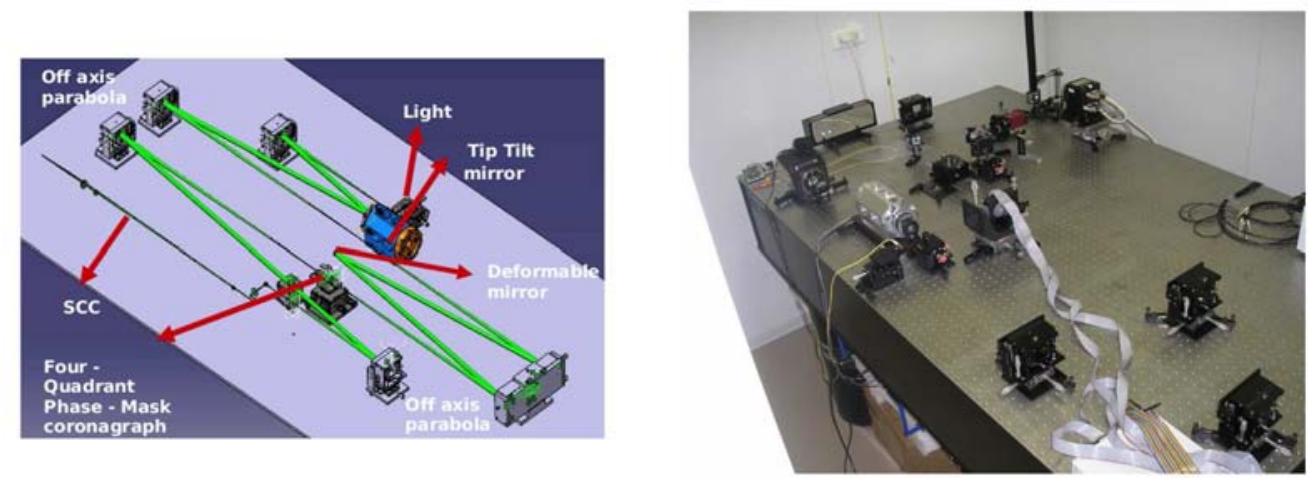

Fig. 4. On the left, a design of the High Contrast Imaging Bench. The detector and the deformable mirror are not represented, the collimated beam diameter is $9 \mathrm{~mm}$ in the deformable mirror plane. On the right, a picture of the bench. At the moment, we use a OKO deformable mirror with 37 actuators. 
In this section we will present the actual bench we have developed to test the SCC principle in laboratory condition. We want to reach a contrast larger than $10^{7}$ on the bench. The main components are a laser, a tip tilt mirror and an electrostatic 32x32 actuators Boston deformable mirror, off axis parabola, a four-quadrant phase mask coronagraph, a Lyot stop and a detector. On Fig. 4, on the left, it is the design of the bench, on the right, it is the actual bench. We first use an electrostatic OKO deformable mirror with 37 actuators in order to test our software.

The light source is a laser diode centered in $\lambda_{0}=635 \mathrm{~nm}$, with a power of $2.5 \mathrm{~mW}$ and a spectral width smaller than $1 \%$. The pupil diameter is determined by the effective zone of the deformable mirror. As the Boston deformable mirror, the OKO mirror is electrostatic, the response of the actuators with the voltage is proportional to $\mathrm{V}^{2}$. The applied voltages can vary between 0 and 160 volts. The diameter of the effective zone is $9 \mathrm{~mm}$. We have calibrated this deformable mirror studying the local deformations of the membrane when we apply a serie of 10 voltages between 0 and 160 volts on each actuator. For each of the 37 actuators, we have checked that the response with the voltage was in $V^{2}$. On Fig. 5, we have represented the response of three actuators located in the middle and on the edge of the membrane of the deformable mirror. This actuators are shown in color on the scheme in Fig. 5. On the top, on the left, it is a picture of the OKO deformable mirror. As we can see, the deformation amplitude is not the same with the location of the actuator. This phenomena is related with the coupling of the mirror. Hence, we have 37 different influence functions. Since the coronagraph is very sensitive to the position of the source, we use a dedicated tip tilt mirror to correct the positionning error. It is composed by two piezoelectric actuators, their maximum stroke is $2 \mathrm{mrad}$ with a precision of $0.16 \mu \mathrm{rad}$.

This bench has just been set up and we are developing tools on labview to drive the tip tilt and the deformable mirrors and to close the adaptative optic loop. However, before to set up this bench, we have done some preliminary experiments to test the SCC coupled with a four-quadrant phase mask coronagraph. We have done a simplified version of the SCC, the bench and the results are described in the paragraph 3.2 .
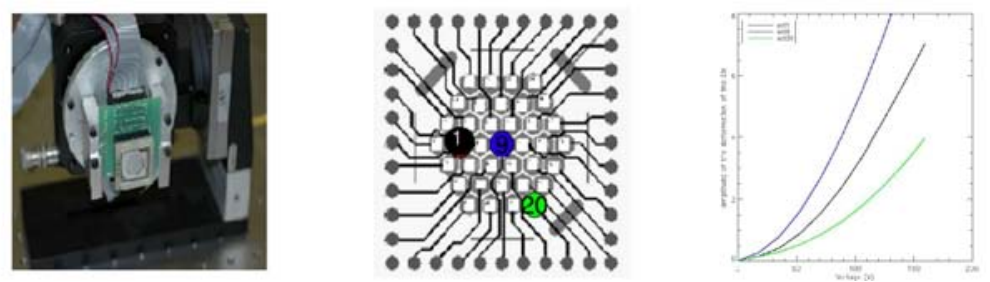

Fig. 5. On the left : a picture of the OKO deformable mirror (DM). In the middle : a scheme of the DM with the location of some actuators. On the right : the graph represents the amplitude deformation of the deformable mirror membrane when applying several voltages on each 37 actuators. The colored curves represent the amplitude deformation of the membrane when voltages are applied on a central actuator and two in the edge (see picture on the left, on the bottom).

\subsection{A simplified SCC prototype}

We have done a simplified version of the SCC as a prototype which is represented on the picture in Fig. 6. The first part of this bench represents the star and is composed by a laser diode (the same as introduce above, in paragraph 3.1) and a lens. Then, a diaphragm is used to simulate the pupil of the telescope. On a third part, there is the four-quadrant phase mask coronagraph. Finally, the last part is the SCC itself. It is composed by a modified Lyot stop, a lens to focus light on the detector, a density wheel to not saturate the detector and a camera. This scheme is different from the one in Fig. 1: the reference channel is created by a modified Lyot stop. This is made of a classical Lyot stop and a small hole located outside of the pupil geometry. Because the coronagraph diffracts most of the stellar light outside of the pupil diameter, the beam which diffracts through this off-axis hole is coherent with stellar light and is bright enough to create fringes in the focal plane. This simple setup for the SCC is 
also described in Galicher et al. 2009 [8]. In Fig. 6, the image in the middle is the Airy disk pattern we got on the detector without neither the coronagraph nor the SCC. On the right, it is the image we got with the SCC coupled with the four-quadrant phase mask coronagraph. On this picture, we can see that the speckles are spatially encoded. With this prototype, the ratio between the brightest pixel with and without coronagraph was around 1400. On this bench, we did not correct neither the turbulence in the laboratory nor the static aberrations. Coupling this prototype with the High Contrast Imaging Bench introduced in the paragraph 3.1, we will study the performence of the SCC using a tip tilt mirror and a deformable mirror to correct static aberrations on the bench.

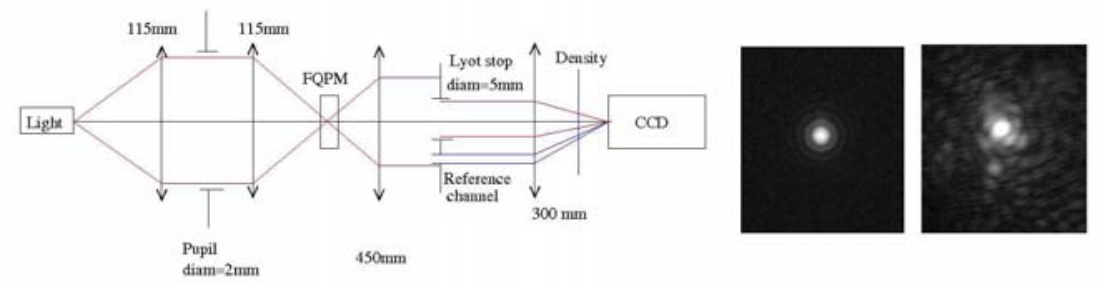

Fig. 6. On the left, simplified version of the SCC. The reference channel is defined in the Lyot stop plane. In the middle, it is the image we get on the detector, without neither a coronagraph nor the SCC. On the right, it is an interferential image obtained with the coronagraph and the SCC.

\section{Conclusion}

The Self-Coherent Camera is studied in the framework of the instrument EPICS for the direct detection of exoplanets. This device is based on coherence to differentiate a true companion from a speckle. With this new device we would be able to reach very high contrast and it could allow us to detect Earth-Like Planet. This instrument could be used either on a spatial-based or ground-based telescope. Numerical validations of the SCC principle coupled with a coronagraph and a deformable mirror for an exposure time shorter than the coherence time of the speckles were done during R. Galicher thesis. In the case of a ground-based telescope, the coherence time of the speckles is very short, hence, we cannot use the SCC with a large signal to noise ratio. We have studied SCC performance with long exposure time. In this case, dynamical phase errors are averaged out unabling the detection of quasi-static aberrations. The behaviour of the SCC working with a long exposure time is under study. We also are working on the experimental validation of the concept both from speckles and faint companion detection.

\section{Acknowledgments}

This work has been supported by : FP7 (Infractructure 2007-1 Grant 211257), la Région Ile de France (Grant SESAME I-06-201/R), INSU/CNRS and l'observatoire de Paris.

\section{References}

1. Kasper, M., Beuzit, J.-L., Verinaud, C., 2009, in this volume.

2. Cavarroc, C., Boccaletti, A., Baudoz, P., Fusco, T., Rouan, D., 2006, A\&A, 447, 397.

3. Baudoz, P., Boccaletti, A., Baudrand, J., Rouan, D., 2006, Proc. of IAU Colloquium.

4. Galicher, R., Baudoz, P., 2007, C. R. Phys., 8, 333.

5. Galicher, R., Baudoz, P., Rousset, G., 2008, A\&A, 488.

6. Consortini, A., Ronchi, L., Moroder, E., 1973, J. Opt. Soc. Am. 63 10, 1246-1248.

7. Baudoz, P., Galicher, R., Mas, M., Rousset, G., 2009, in this volume.

8. Galicher, R., Baudoz, P., Rousset, G., Totems, J., 2009, accepted in A\&A. 